\title{
GAIA Insufficient Evidence for Diagnosis of Preeclampsia with Severe Features
}

National Cancer Institute

\section{Source}

National Cancer Institute. GAIA Insufficient Evidence for Diagnosis of Preeclampsia with

Severe Features. NCl Thesaurus. Code C128008.

GAIA Insufficient Evidence for Diagnosis of Preeclampsia with Severe Features is a pregnancy with a gestational age greater than or equal to 20 weeks and the inability to measure blood pressure. 